\title{
Olfactory bulb $\alpha$-synucleinopathy has high specificity and sensitivity for Lewy body disorders
}

\author{
Thomas G. Beach \\ Sun Health Research Institute, 10515 West Santa Fe Drive, Sun City, AZ 85351, USA
}

Charles L. White III and Christa L. Hladik

University of Texas Southwestern Medical Center, Dallas, TX, USA

Marwan N. Sabbagh, Donald J. Connor, Holly A. Shill, Lucia I. Sue, Jeanne Sasse, Jyothi

Bachalakuri, and Jonette Henry-Watson

Sun Health Research Institute, 10515 West Santa Fe Drive, Sun City, AZ 85351, USA

\author{
Haru Akiyama \\ Tokyo Institute of Psychiatry, Tokyo, Japan \\ Charles H. Adler \\ Mayo Clinic, Scottsdale, AZ, USA

\section{The Arizona Parkinson's Disease Consortium}

\section{Abstract}

\begin{abstract}
Involvement of the olfactory bulb by Lewy-type $\alpha$-synucleinopathy (LTS) is known to occur at an early stage of Parkinson's disease (PD) and Lewy body disorders and is therefore of potential usefulness diagnostically. An accurate estimate of the specificity and sensitivity of this change has not previously been available. We performed immunohistochemical $\alpha$-synuclein staining of the olfactory bulb in 328 deceased individuals. All cases had received an initial neuropathological examination that included $\alpha$-synuclein immunohistochemical staining on sections from brainstem, limbic and neocortical regions, but excluded olfactory bulb. These cases had been classified based on their clinical characteristics and brain regional distribution and density of LTS, as PD, dementia with Lewy bodies (DLB), Alzheimer's disease with LTS (ADLS), Alzheimer's disease without LTS (ADNLS), incidental Lewy body disease (ILBD) and elderly control subjects. The numbers of cases found to be positive and negative, respectively, for olfactory bulb LTS were: PD 55/3; DLB 34/1; ADLS 37/5; ADNLS 19/84; ILBD 14/7; elderly control subjects 5/64. The sensitivities and specificities were, respectively: 95 and $91 \%$ for PD versus elderly control; 97 and $91 \%$ for DLB versus elderly control; 88 and $91 \%$ for ADLS versus elderly control; 88 and $81 \%$ for ADLS versus ADNLS; 67 and $91 \%$ for ILBD versus elderly control. Olfactory bulb synucleinopathy density scores correlated significantly with synucleinopathy scores in all other brain regions (Spearman $R$ values between 0.46 and 0.78) as well as with scores on the Mini-Mental State Examination and Part 3 of the unified Parkinson's Disease Rating Scale (Spearman $R-0.27,0.35$, respectively). It is concluded that olfactory bulb LTS accurately predicts the presence of LTS in other brain regions. It is suggested that olfactory bulb biopsy be considered to confirm the diagnosis in PD subjects being assessed for surgical therapy.
\end{abstract}

e-mail: thomas.beach@bannerhealth.org; thomas.beach@sunhealth.org

Conflict of interest statement All authors have declared that they have no competing interests. 


\section{Keywords}

Parkinson's disease, surgery; Deep brain stimulation; Gene therapy; Transplantation; Dementia with Lewy bodies, diagnosis, therapy, clinical trial; $\alpha$-Synuclein, Lewy bodies, incidental Lewy body disease; Biopsy; Olfactory bulb

\section{Introduction}

It has long been observed that subjects with Parkinson's disease (PD) have decreased olfactory sensation $[1,10,17,24]$. Attempts to use tests of olfaction for clinical diagnosis have had mixed results, however, due to loss of olfactory ability in other conditions as well [22,32]. Lewy bodies and synucleinopathy have been reported to be present in the olfactory bulbs of subjects with PD and dementia with Lewy bodies (DLB) as well as asymptomatic subjects with incidental Lewy body disease (ILBD) [5,7,9,19,35], suggesting that olfactory bulb synucleinopathy is present in several Lewy body disorders and occurs at an early stage of disease. Additionally, synucleinopathy and tauopathy have been reported to co-exist in affected olfactory bulb neurons of subjects with Alzheimer's disease [11].

The presence of synucleinopathy in the olfactory bulb makes it theoretically possible to improve upon clinical diagnostic accuracy for Lewy body disorders during life, using olfactory bulb biopsy. However, the specificity and sensitivity of ollfactory bulb synucleinopathy should be established in a large autopsy series. To achieve this, we undertook immunohistochemical $\alpha$-synuclein staining of the olfactory bulb in 328 autopsied elderly individuals.

\section{Materials and methods}

\section{Human subjects}

The study took place at Sun Health Research Institute (SHRI), which is a non-profit organization located in the Sun Cities retirement communities of northwest metropolitan Phoenix, AZ. Sun Health Research Institute and the Mayo Clinic Arizona are the principal members of the Arizona Parkinson's Disease Consortium. Brain necropsies and neuropathological examinations were performed on elderly subjects who had volunteered for the SHRI Brain Donation Program [3]. The Brain Donation Program has been approved by the Institutional Review Board of Sun Health Research Institute. Subjects were chosen by searching the Brain Donation Program Database for all those that died between 1 January 1997 and 31 December 2006 with clinicopathologic diagnoses of control (no clinical history of dementia or parkinsonism), PD, DLB and AD, for whom olfactory bulb tissue was available and for whom a complete set of brain blocks had been stained immunohistochemically for $\alpha$ synuclein. The brain regions were chosen based on published staging procedures for Parkinson's disease [6] and dementia with Lewy bodies [30] and therefore included anterior medulla, anterior pons, midbrain, amygdala, cingulate gyrus, middle temporal gyrus, middle frontal gyrus and inferior parietal lobule.

Subjects received standardized neuropathological examinations as described previously [3]. Specific consensus diagnostic criteria were used for Alzheimer's disease (AD) [40], Parkinson's disease [13] and dementia with Lewy bodies [31]. For both AD and DLB, cases received the diagnosis if they were classified as "intermediate" or "high" probabilities in their respective classification schemes. Cases with Lewy-type synucleinopathy but not meeting these diagnostic criteria were designated as either incidental Lewy body disease (ILBD) or Alzheimer's disease with Lewy-type synucleinopathy (ADLS). 
All subjects were clinically characterized by review of medical records, self-report and interviews with spouses and/or caregivers. As part of the Brain Donation Program's standard protocol, 2 years of private medical records are obtained from the subjects' private physicians, both at the time of enrollment and at the time of death. A subset of subjects received standardized neuropsychological and movement disorder assessments at SHRI, including the Mini Mental State Examination (MMSE) and unified Parkinson's Disease Rating Scale (UPDRS).

\section{Tissue processing and analysis}

Paraffin-embedded blocks of anterior medulla, anterior pons, amygdala, cingulate gyrus, middle frontal gyrus, middle temporal gyrus and inferior parietal lobule were initially stained after brain necropsy using an immunoperoxidase method for $\alpha$-synuclein. Sections were stained after pretreatment with proteinase K [18] using an antiserum raised against an $\alpha$-synuclein peptide fragment that was phosphorylated at serine 129 [12]. Color development was achieved with an avidin-biotin-horseradish peroxidase complex (ABC) and 3,3'-diaminobenzidine enhanced with nickel ammonium sulfate; the method has been generally described in a prior publication [4]. The same method was later used to stain paraffin-embedded olfactory bulb sections from the 328 cases, for the present study. Lewy bodies and associated neurites in the substantia nigra were identified in $40 \mu \mathrm{m}$ sections of midbrain stained with thioflavine S. Lewy bodies and associated neurite density were graded in all brain regions as mild, moderate, severe and very severe, according to the templates published by the Dementia with Lewy Bodies Consortium [30].

\section{Statistical analysis}

Statistical analyses consisted of Spearman correlation of olfactory bulb synucleinopathy scores with synucleinopathy scores in other brain regions as well as with MMSE and motor UPDRS scores. Logistic regression analysis was used to obtain odds ratios as well as sensitivities and specificities. For the latter determinations, the dependent binary variable in all cases was diagnosis while the sole independent variable was defined by positive or negative evidence of synucleinopathy in the olfactory bulb.

\section{Results}

\section{Description of diagnostic groups}

Of the 328 subjects with a full set of paraffin blocks available, there were 69 elderly controls, 21 ILBD, 58 PD, 35 DLB, 42 ADLS and 103 AD without synucleinopathy (ADNLS), based on prior evaluations not including examination of the olfactory bulb (Table 1). The subjects ranged in age from 64 to 103, with mean ages for the diagnostic groups ranging from 79.7 (PD) to 85.7 (ILBD). Of the DLB cases, all but four also fulfilled diagnostic criteria for AD. The cases with combined DLB/AD were classified, for the purposes of this study, only as DLB and were not included in the AD groups.

\section{Morpholologic characteristics of olfactory bulb synucleinopathy}

In cases showing positive immunostaining for $\alpha$-synuclein, the stained elements usually resembled neuronal perikaryal cytoplasmic inclusions or neuritic fibers, while a smaller fraction were dot-like, possibly representing presynaptic terminals or fibers cut in cross-section (Fig. 1a-f). In the great majority of cases, both perikaryal cytoplasmic inclusions and fibers were present. Rarely, bulbs contained only perikaryal cytoplasmic inclusions or only neurites. Perikaryal inclusions were mostly in the form of round or irregular areas of cytoplasmic staining. Inclusions resembling classical Lewy bodies, with a distinguishable core-and-halo appearance, were only seen in approximately $10 \%$ of cases (Fig. 1d). The neurites were mostly 
fine and unbranching, with occasional enlarged distorted forms (Fig. 1e) that were similar to Lewy-related neurites reported for other brain regions. The bulb region most frequently and heavily involved was the anterior olfactory nucleus, consisting of multipolar medium-sized neurons (Fig. 1a-d), arranged in small discontinuous groups along the central axis of the bulb. The inner plexiform layer (Fig. 1f) was the next most frequently and heavily affected area and the staining in this layer was always neuritic, without perikaryal inclusions. It is noted here that our usage of the term "anterior olfactory nucleus" is restricted to those neuronal groups that reside within the bulb itself. We did not examine the nucleus of the same name that is located near the ventral brain surface at the junction of the olfactory tract with the anterior perforated substance.

\section{Sensitivity and specificity of olfactory bulb synucleinopathy for Lewy body disorders}

The presence of synucleinopathy in the olfactory bulb was highly predictive of synucleinopathy elsewhere in the brain, regardless of the diagnostic classification of cases. The numbers of cases, by diagnosis, found to be positive and negative, respectively, for olfactory bulb Lewytype synucleinopathy were PD 55/3, DLB 34/1, ADLS 37/5, ADNLS 19/84; elderly control subjects without diagnoses of parkinsonism, dementia or ILBD 5/64, ILBD 14/7. Olfactory bulb staining revealed 24 cases (19 among Alzheimer's disease cases, 5 among the elderly control group) that were previously unsuspected to be Lewy body disorders, as brain sections from other regions in these cases had all been negative for $\alpha$-synuclein. The sensitivities and specificities for specific diagnostic categories (Table 2) were 95\% sensitive, 93\% specific for the diagnosis of PD versus elderly control; $97 \%$ sensitive, $93 \%$ specific for DLB versus elderly control; 88\% sensitive, 93\% specific for ADLS versus control; 88\% sensitive, $81 \%$ specific for ADLS versus ADNLS; $71 \%$ sensitive, $93 \%$ specific for ILBD versus control. The results were all statistically significant (Wald $P<0.00001$ ).

\section{Correlation of olfactory bulb synucleinopathy score with those in other brain regions}

Correlation analysis (Table 3 ) showed that olfactory bulb synucleinopathy density scores correlated significantly with those in other brain regions, with Spearman correlation coefficients ranging from 0.46 (inferior parietal lobule) to 0.78 (amygdala). All correlations were statistically significant (Spearman $P<0.0001$ ).

\section{Correlation of olfactory bulb synucleinopathy score with MMSE and UPDRS scores}

Correlation analysis (Table 3 ) showed that olfactory bulb synucleinopathy density scores correlated significantly with MMSE and UPDRS (motor part) scores, with Spearman correlation coefficients of -0.27 and 0.35 , respectively. Both correlations were statistically significant (Spearman $P<0.0001$ ).

\section{Discussion}

\section{Olfactory bulb synucleinopathy accurately predicts the presence of Lewy body disorders}

The results of this investigation show that the presence of synucleinopathy in the olfactory bulb predicts, with greater than $90 \%$ sensitivity and specificity, the existence of neuropathologically confirmed PD and DLB. Additionally, olfactory bulb synucleinopathy predicts with high accuracy the presence of Lewy body pathology in subjects with $\mathrm{AD}$. It is probable that olfactory bulb synucleinopathy occurs at early stages of Lewy body disorders as it is present in the majority of cases of ILBD, the suspected preclinical form of PD and/or DLB [2]. These results also show that the severity of olfactory bulb synucleinopathy may serve as a rough guage of the severity of Lewy-related pathology throughout the CNS, as it correlates with the severity of synucleinopathy in the brainstem, limbic regions and neocortex, as well as with measures of the severity of cognitive and motor dysfunction. While it has previously been reported that 
the olfactory bulb is involved at early stages of Lewy body disorders [7,19], the sensitivity and specificity of olfactory bulb synucleinopathy for such conditions had not been estimated.

\section{Olfactory bulb biopsy: possible indication for patient selection for PD surgical therapy}

Until now, there has not been a practical and ethical option for a biopsy diagnosis of PD. Cortical biopsy has been performed in the past for Alzheimer's disease [16] but has generally been discontinued for ethical reasons [28], as the harm caused by the procedure cannot be offset by benefits arising from accurate diagnosis. The possible usage of clinical olfactory testing as a predictive risk factor for PD has also been critiqued [26]. Pharmacologic therapy of both Alzheimer's disease and Parkinson's disease is initiated empirically as the risks of therapy are low and therapy can be discontinued if it is ineffective or causes adverse effects. Surgical treatment of PD, however, which has included pallidotomy, thalamotomy, deep brain stimulation and neural transplantation, carries significant risk of complications, up to $40 \%$ overall $[15,25,29,33,34,36-39]$. These therapies are thought to be ineffective for non-PD causes of Parkinsonism and therefore accurate diagnosis of PD is considered essential for patient selection. Estimates of the current clinical accuracy of the diagnosis of PD range between 65 and $90 \%[8,13,14,20,21,27]$, with the higher accuracy figures being dependent on prolonged clinical observation and therapeutic trial with levodopa. Five years of observation with a favorable response to levodopa is recommended for surgical PD candidates [25]. It has been reported that patients with multiple system atrophy (MSA) misdiagnosed as PD have undergone placement of deep brain stimulators and have not had a lasting benefit [25]. As the characteristic glial cytoplasmic synuclein-immunoreactive inclusions of MSA are also present and diagnostic in the olfactory bulb, as reported by Kovacs et al. [23] (we have confirmed this finding in five MSA cases), olfactory bulb biopsy would differentiate between PD and MSA. Olfactory bulb biopsy might therefore be useful for the evaluation of candidates for surgical therapy of PD, where the risks of biopsy might be justified if it would spare non-PD subjects the greater risks associated with pallidotomy, thalamotomy, deep brain stimulation or neural transplantation [15,25,29,33,34,36-39].

\section{Limitations of the current study}

As this was an autopsy study, the entire olfactory bulb was available for examination. To achieve similar results in a biopsy setting, it is therefore recommended that the entire bulb be removed and examined. Examination of only part of the bulb may give false-negative results in some cases due to localized synucleinopathy.

\section{Conclusions}

The presence of Lewy-type $\alpha$-synucleinopathy in the olfactory bulb has greater than $90 \%$ sensitivity and specificity for neuropathologically confirmed PD and DLB and its severity correlates with the severity of synucleinopathy in other brain regions as well as with measures of cognition and motor function. Olfactory bulb biopsy might be considered in the setting of patient evaluation for surgical therapy for PD.

\section{Acknowledgments}

We wish to acknowledge all of the other members of the Arizona Parkinson's Disease Consortium, including John Caviness MD, Virgilio Evidente MD, Erika Driver-Dunckley MD, Padma Mahant MD, and Johan Samanta MD. This research is supported by grants to the Sun Health Research Institute Brain Donation Program and the Arizona Parkinson's Disease Consortium by the Michael J. Fox Foundation for Parkinson's Research (The Prescott Family Initiative), the Arizona Biomedical Research Commission (contracts 4001, 0011 and 05-901) and the National Institute on Aging (P30 AG19610). 


\section{References}

1. Adler CH. Nonmotor complications in Parkinson's disease. Mov Disord 2005;20(Suppl 11):S23-S29. [PubMed: 15822106]

2. Beach TG, Adler CH, Sue LI, Peirce JB, Bachalakuri J, sing-Hernandez JE, Lue LF, Caviness JN, Connor DJ, Sabbagh MN, Walker DG. Reduced striatal tyrosine hydroxylase in incidental Lewy body disease. Acta Neuropathol 2008;115:445-451. [PubMed: 17985144]

3. Beach TG, Sue LI, Walker DG, Roher AE, Lue L, Vedders L, Connor DJ, Sabbagh MN, Rogers J. The Sun Health Research Institute Brain Donation Program: description and experience, 1987-2007. Cell Tissue Bank. 2007

4. Beach TG, Tago H, Nagai T, Kimura H, McGeer PL, McGeer EG. Perfusion-fixation of the human brain for immunohistochemistry: comparison with immersion-fixation. J Neurosci Methods 1987;19:183-192. [PubMed: 2437408]

5. Bloch A, Probst A, Bissig H, Adams H, Tolnay M. Alpha-synuclein pathology of the spinal and peripheral autonomic nervous system in neurologically unimpaired elderly subjects. Neuropathol Appl Neurobiol 2006;32:284-295. [PubMed: 16640647]

6. Braak H, Bohl JR, Muller CM, Rub U, de Vos RA, Del TK. Stanley Fahn Lecture 2005: the staging procedure for the inclusion body pathology associated with sporadic Parkinson's disease reconsidered. Mov Disord 2006;21:2042-2051. [PubMed: 17078043]

7. Braak H, Del Tredici K, Bratzke H, Hamm-Clement J, Sandmann-Keil D, Rub U. Staging of the intracerebral inclusion body pathology associated with idiopathic Parkinson's disease (preclinical and clinical stages). J Neurol 2002;249(Suppl 3):1-5. [PubMed: 11954855]

8. Cornford ME, Chang L, Miller BL. The neuropathology of Parkinsonism: an overview. Brain Cogn 1995;28:321-341. [PubMed: 8546858]

9. Daniel SE, Hawkes CH. Preliminary diagnosis of Parkinson's disease by olfactory bulb pathology. Lancet 1992;340:186. [PubMed: 1352606]

10. Doty RL, Deems DA, Stellar S. Olfactory dysfunction in Parkinsonism: a general deficit unrelated to neurologic signs, disease stage, or disease duration. Neurology 1988;38:1237-1244. [PubMed: 3399075]

11. Fujishiro H, Tsuboi Y, Lin WL, Uchikado H, Dickson DW. Co-localization of tau and alpha-synuclein in the olfactory bulb in Alzheimer's disease with amygdala Lewy bodies. Acta Neuropathol 2008;116:17-24. [PubMed: 18446351]

12. Fujiwara H, Hasegawa M, Dohmae N, Kawashima A, Masliah E, Goldberg MS, Shen J, Takio K, Iwatsubo T. Alpha-Synuclein is phosphorylated in synucleinopathy lesions. Nat Cell Biol 2002;4:160-164. [PubMed: 11813001]

13. Gelb DJ, Oliver E, Gilman S. Diagnostic criteria for Parkinson disease. Arch Neurol 1999;56:33-39. [PubMed: 9923759]

14. Gibb WR. Neuropathology of Parkinson's disease and related syndromes. Neurol Clin 1992;10:361376. [PubMed: 1584179]

15. Gill CE, Konrad PE, Davis TL, Charles D. Deep brain stimulation for Parkinson's disease: the Vanderbilt University Medical Center experience, 1998-2004. Tenn Med 2007;100:45-47. [PubMed: 17474384]

16. Harbaugh RE. Brain biopsy in Alzheimer's disease: surgical technique and indications. Bull Clin Neurosci 1985;50:39-43. [PubMed: 3842084]

17. Hawkes C. Olfaction in neurodegenerative disorder. Adv Otorhinolaryngol 2006;63:133-151. [PubMed: 16733338]

18. Hladik CL, White CL. Comparison of digestive enzyme and formic acid pretreatment for optimal immunohistochemical demonstration of alpha-synuclein-immunoreactive cerebral cortical Lewy neurites. J Neuropathol Exp Neurol 2003;62:554.

19. Hubbard PS, Esiri MM, Reading M, McShane R, Nagy Z. Alpha-synuclein pathology in the olfactory pathways of dementia patients. J Anat 2007;211:117-124. [PubMed: 17553102]

20. Hughes AJ, Daniel SE, Kilford L, Lees AJ. Accuracy of clinical diagnosis of idiopathic Parkinson's disease: a clinico-pathological study of 100 cases. J Neurol Neurosurg Psychiatry 1992;55:181-184. [PubMed: 1564476] 
21. Hughes AJ, Daniel SE, Lees AJ. Improved accuracy of clinical diagnosis of Lewy body Parkinson's disease. Neurology 2001;57:1497-1499. [PubMed: 11673599]

22. Katzenschlager R, Lees AJ. Olfaction and Parkinson's syndromes: its role in differential diagnosis. Curr Opin Neurol 2004;17:417-423. [PubMed: 15247536]

23. Kovacs T, Papp MI, Cairns NJ, Khan MN, Lantos PL. Olfactory bulb in multiple system atrophy. Mov Disord 2003;18:938-942. [PubMed: 12889086]

24. Kranick SM, Duda JE. Olfactory dysfunction in Parkinson's disease. Neurosignals 2008;16:35-40. [PubMed: 18097158]

25. Lang AE, Houeto JL, Krack P, Kubu C, Lyons KE, Moro E, Ondo W, Pahwa R, Poewe W, Troster AI, Uitti R, Voon V. Deep brain stimulation: preoperative issues. Mov Disord 2006;21(Suppl 14):S171-S196. [PubMed: 16810718]

26. Linazasoro G. Olfaction testing in PD: risky conclusions. Mov Disord 2008;23:1060. [PubMed: 17557341]

27. Litvan I, MacIntyre A, Goetz CG, Wenning GK, Jellinger K, Verny M, Bartko JJ, Jankovic J, McKee A, Brandel JP, Chaudhuri KR, Lai EC, D'Olhaberriague L, Pearce RK, Agid Y. Accuracy of the clinical diagnoses of Lewy body disease, Parkinson disease, and dementia with Lewy bodies: a clinicopathologic study. Arch Neurol 1998;55:969-978. [PubMed: 9678315]

28. Mahendra B. Some ethical issues in dementia research. J Med Ethics 1984;10:29-31. [PubMed: 6231378]

29. Master Z, McLeod M, Mendez I. Benefits, risks and ethical considerations in translation of stem cell research to clinical applications in Parkinson's disease. J Med Ethics 2007;33:169-173. [PubMed: 17329391]

30. McKeith IG, Dickson DW, Lowe J, Emre M, O'Brien JT, Feldman H, Cummings J, Duda JE, Lippa C, Perry EK, Aarsland D, Arai H, Ballard CG, Boeve B, Burn DJ, Costa D, Del ST, Dubois B, Galasko D, Gauthier S, Goetz CG, Gomez-Tortosa E, Halliday G, Hansen LA, Hardy J, Iwatsubo T, Kalaria RN, Kaufer D, Kenny RA, Korczyn A, Kosaka K, Lee VM, Lees A, Litvan I, Londos E, Lopez OL, Minoshima S, Mizuno Y, Molina JA, Mukaetova-Ladinska EB, Pasquier F, Perry RH, Schulz JB, Trojanowski JQ, Yamada M. Diagnosis and management of dementia with Lewy bodies: third report of the DLB Consortium. Neurology 2005;65:1863-1872. [PubMed: 16237129]

31. McKeith IG, Galasko D, Kosaka K, Perry EK, Dickson DW, Hansen LA, Salmon DP, Lowe J, Mirra SS, Byrne EJ, Lennox G, Quinn NP, Edwardson JA, Ince PG, Bergeron C, Burns A, Miller BL, Lovestone S, Collerton D, Jansen EN, Ballard C, de Vos RA, Wilcock GK, Jellinger KA, Perry RH. Consensus guidelines for the clinical and pathologic diagnosis of dementia with Lewy bodies (DLB): report of the consortium on DLB international workshop. Neurology 1996;47:1113-1124. [PubMed: 8909416]

32. McKinnon JH, Demaerschalk BM, Caviness JN, Wellik KE, Adler CH, Wingerchuk DM. Sniffing out Parkinson disease: can olfactory testing differentiate Parkinsonian disorders? Neurologist 2007;13:382-385. [PubMed: 18090718]

33. Obeso JA, Rodriguez MC, Gorospe A, Guridi J, Alvarez L, Macias R. Surgical treatment of Parkinson's disease. Baillieres Clin Neurol 1997;6:125-145. [PubMed: 9426872]

34. Paluzzi A, Belli A, Bain P, Liu X, Aziz TM. Operative and hardware complications of deep brain stimulation for movement disorders. Br J Neurosurg 2006;20:290-295. [PubMed: 17129876]

35. Pearce RK, Hawkes CH, Daniel SE. The anterior olfactory nucleus in Parkinson's disease. Mov Disord 1995;10:283-287. [PubMed: 7651444]

36. Rodriguez RL, Fernandez HH, Haq I, Okun MS. Pearls in patient selection for deep brain stimulation. Neurologist 2007;13:253-260. [PubMed: 17848865]

37. Seijo FJ, varez-Vega MA, Gutierrez JC, Fdez-Glez F, Lozano B. Complications in subthalamic nucleus stimulation surgery for treatment of Parkinson's disease. Review of 272 procedures. Acta Neurochir (Wien) 2007;149:867-875. [PubMed: 17690838]

38. Subramanian T. Cell transplantation for the treatment of Parkinson's disease. Semin Neurol 2001;21:103-115. [PubMed: 11346020]

39. Tir M, Devos D, Blond S, Touzet G, Reyns N, Duhamel A, Waucquier N, Cottencin O, Dujardin K, Debailleul AM, Cassim F, Destee A, Defebvre L, Krystkowiak P. Exhaustive, one-year follow-up 
of subthalamic nucleus deep brain stimulation in a large, single-center cohort of parkinsonian patients. Neurosurgery. 2007

40. The National Institute on Aging; Reagan Institute Working Group on Diagnostic Criteria for the Neuropathological Assessment of Alzheimer's Disease. Consensus recommendations for the postmortem diagnosis of Alzheimer's disease. Neurobiol Aging 1997;18:S1-S2. [PubMed: 9330978] 

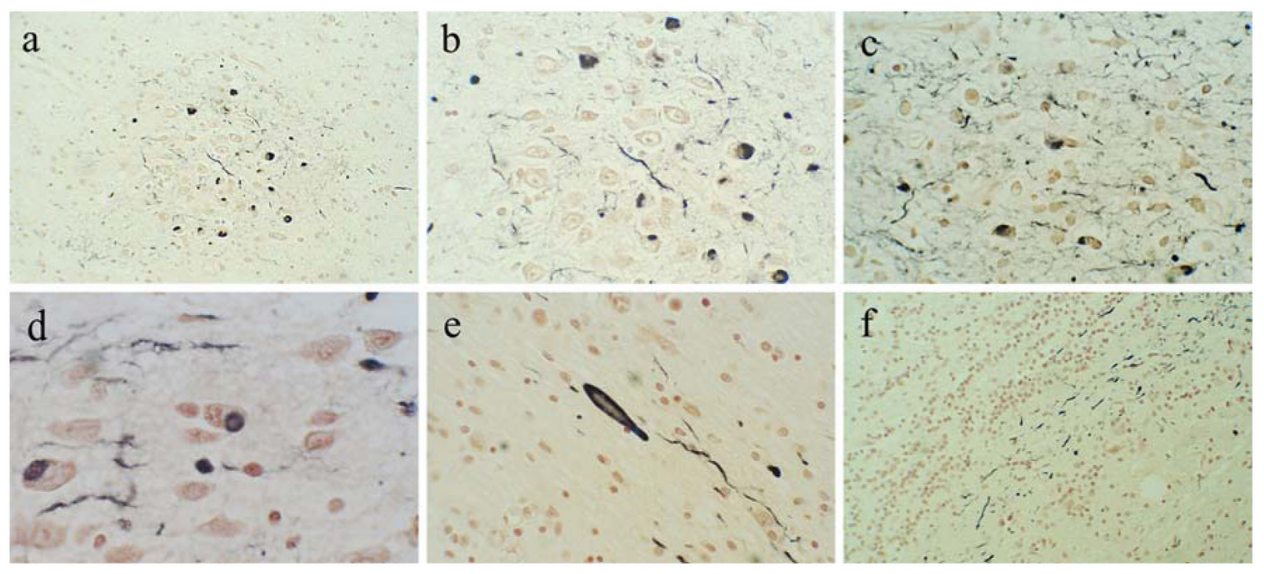

Fig. 1.

Olfactory bulb sections depicting $\alpha$-synuclein-immunoreactive features. a Low-magnification photomicrograph of the anterior olfactory nucleus within the olfactory bulb; both neuronal perikaryal cytoplasmic inclusions and neurites are stained. b Higher-magnification image of anterior olfactory nucleus from previous panel. c Anterior olfactory nucleus from another subject, again illustrating both neuronal perikaryal cytoplasmic inclusions and neurites. $\mathbf{d}$ Lewy body-like inclusion (center) within a neuron of the anterior olfactory nucleus. e Swollen, dystrophic neurite within the olfactory tract, immunoreactive for $\alpha$-synuclein, $f$ Fibers immunoreactive for $\alpha$-synuclein, coursing within the internal plexiform layer 
Table 1

Age and neuropathologic diagnoses of study subjects

\begin{tabular}{|c|c|c|c|}
\hline Diagnosis & $N$ & $\begin{array}{l}\text { Mean age } \\
\text { (SD) }\end{array}$ & Range \\
\hline Elderly control (EC) & 69 & $86.0(5.64)$ & $73-100$ \\
\hline Incidental Lewy body disease (ILBD) & 21 & $85.7(6.33)$ & $73-103$ \\
\hline Parkinson's disease (PD) & 58 & $79.7(6.26)$ & $64-90$ \\
\hline Dementia with Lewy bodies $^{a}$ (DLB) & 35 & $81.4(7.34)$ & $64-95$ \\
\hline Alzheimer's disease with & 42 & $83.5(7.76)$ & $66-102$ \\
\hline \multicolumn{4}{|l|}{ Lewy synucleinopathy (ADLS) } \\
\hline Alzheimer's disease without & 103 & $83.5(8.73)$ & $48-98$ \\
\hline Lewy synucleinopathy (ADNLS) & & & \\
\hline
\end{tabular}

Values shown are mean values, with standard deviations in parentheses

${ }^{a}$ All but four cases also met diagnostic criteria for AD 
Table 2

Odds ratios, $95 \%$ confidence intervals, sensitivity and specificity for olfactory bulb synucleinopathy by diagnostic category

\begin{tabular}{lllrr}
\hline Comparison & OR $(\mathbf{9 5} \% \mathbf{C I})$ & Sensitivity $(\boldsymbol{\%})$ & Specificity $(\boldsymbol{\%})$ \\
\hline PD versus EC & $572(65-5,065)$ & 98 & 91 & $<0.000001$ \\
DLB versus EC & $378(42-3,373)$ & 97 & 91 & $<000001$ \\
ADLS versus EC & $83(22-305)$ & 89 & 81 & 85 \\
ADLS versus ADNLS & $44(15-126)$ & 89 & 91 & $<0.000001$ \\
ILBD versus EC & $22(6-78)$ & 69 & $<000001$ \\
\hline
\end{tabular}

See Table 1 for abbreviations 


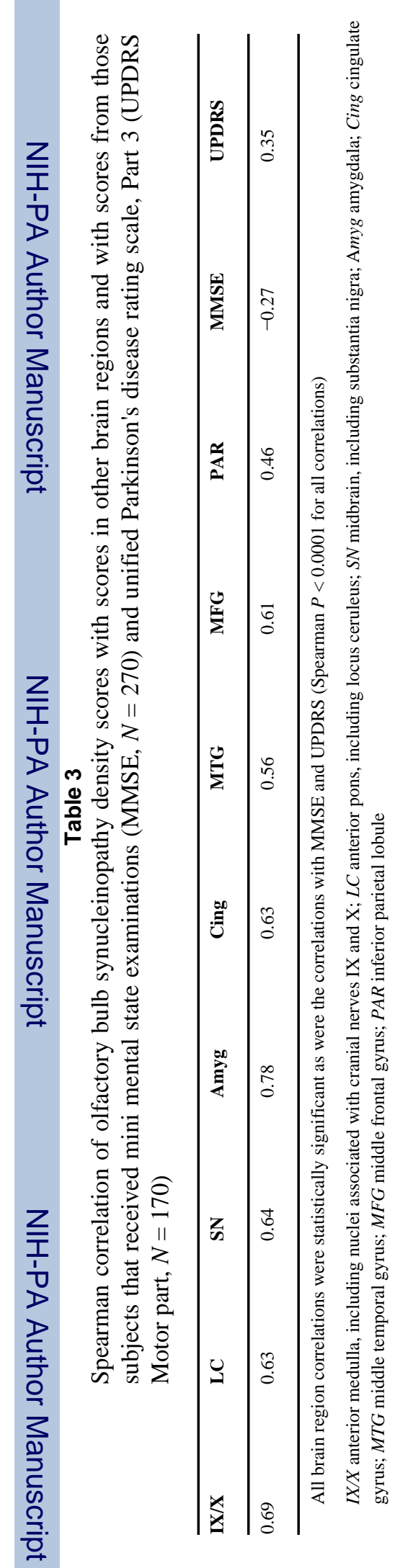

Acta Neuropathol. Author manuscript; available in PMC 2010 February 1. 